
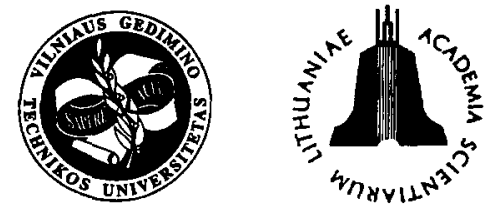

JOURNAL OF CIVIL ENGINEERING AND MANAGEMENT

http:/www.vtu.lt/english/edition

2002, Vol VIII, No 1, 68-72

\title{
DAMAGE-INDUCED STRESS-SOFTENING EFFECTS IN ELASTOMERIC AND BIOLOGICAL MEMBRANES
}

\author{
Rasa Kazakevičiūtẻ-Makovska \\ Dept of Strength of Materials, Vilnius Gediminas Technical University, \\ Sauletekio al. 11, LT-2040 Vilnius, Lithuania.E-mail: rasa.kama@st.vtu.lt
}

Received 27 Dec 2001; accepted 10 Febr 2002

\begin{abstract}
The two-dimensional finite strain constitutive model for membranes is presented; it incorporates stress-softening behaviour typically observed in elastomeric and natural or biologically-derived soft membranes subjected to severe deformations. It is assumed that the experimentally observed progressive degradation of a membrane stiffness under monotonous and cycling loading can macroscopically be modelled by a scalar damage variable. The evolution of this variable during the deformation process is specified by the kinetic law of damage growth, which together with the constitutive equation for the surface stress tensor and the damage criteria completely determines the presented constitutive model. It is shown that the general constitutive model can be specified for particular classes of problems under certain additional assumptions. In particular, a remarkable simplification of the model is achieved assuming that the state of strain at membrane points can be characterised by a single scalar variable, the so-called effective (equivalent) strain. This assumption is combined with the hypothesis of maximum strain according to which the stress softening in the membrane depends only on the maximum previous strain experienced during deformation history. Within these two hypotheses the progressive degradation of membrane stiffness is completely described by a softening function which determines the current value of damage variable in terms of maximum equivalent strain. Various specific forms of such a softening function as well as different definitions of the effective strain are considered.
\end{abstract}

Keywords: damage mechanics, elastomeric membranes, biological membranes, constitutive equations.

\section{Introduction}

Membranes are widely used as structural elements in many engineering fields. Fabric (pneumatic and tension) structures, automobile airbags, parachutes, meteorological balloons are examples. Membranes have also their natural appearance, eg soap films and bubbles, biomembranes and fluid films. One of the difficulties in modelling membranes is to account for their highly nonlinear elastic and inelastic response to external loading.

While not a problem of great technological importance, the inflation of a toy balloon provides a good illustration of a highly non-linear behaviour of elastomeric membranes. When a balloon is inflated, its radius increases continually until the internal pressure reaches a maximum at a relatively small deformation. If the balloon is further inflated, the pressure decreases, and later rises again at very large strains. This behaviour of a balloon results from combined effects of geometric and material non-linearities and it is also observed in inflating balloons of other shapes. Over years, the problem of inflation of membranes has been studied by many authors under the assumption that the deformation is completely reversible. However, the perfect elasticity is often a very crude assumption.

A typical inelastic effect observed in the inflation and subsequent deflation of balloons is that of hysteresis accompanied by stress-softening (Fig 1). These effects are also observed in biological membranes. However, un-

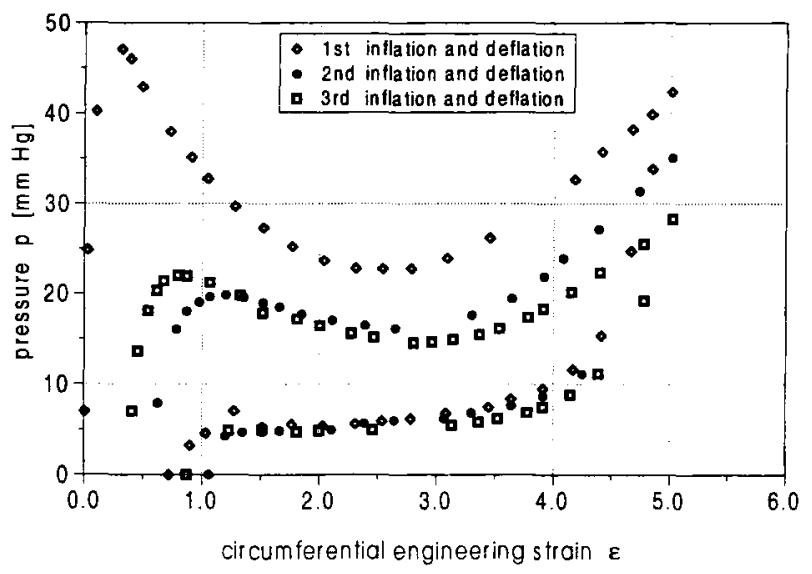

Fig 1. Experimental study of latex balloon inflation/deflation [1] 
like the polymeric membranes, they display highly anisotropic behaviour. Also, stress-softening in such membranes is anisotropic; a load in one direction does not produce the same degree of softening when applied in a different direction (Fig 2).

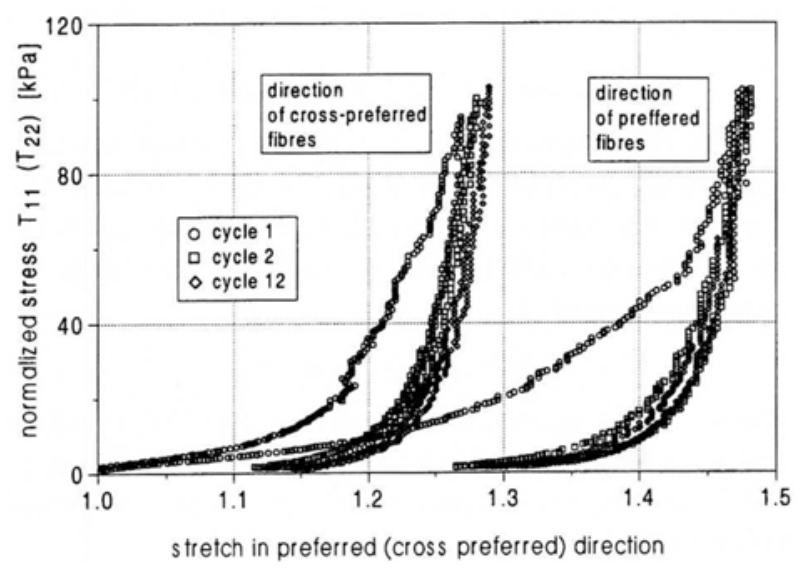

Fig 2. Biaxial experimental tests on rat bladder wall [2]

Elastomeric and biological membranes are often modelled as elastic or viscoelastic ones (eg [3-6]). However, standard elastic and viscoelastic formulations are not able to predict the damage-induced stress-softening effects. In polymeric materials and membranes, this effect is originated by internal damage in the form of progressive debonding of polymer chains. Physical mechanisms underlying stress-softening in biological membranes is more complex and actually poorly understood (cf. $[2,7$, 8]). Nevertheless, phenomenological modelling using twodimensional constitutive equations is possible and often very successful.

The aim of this work is to develop a constitutive model which is capable to capture main effects of damage-induced stress-softening observed in polymeric and biological membranes. This goal is achieved through the use of certain concepts from the field of continuum damage mechanics. As a final result, a simple description of stress-softening effects is derived through successive specification of the general constitutive equations.

\section{Notation and basic equations}

In the coordinate free description (absolute tensor notation), the geometry, kinematics and dynamics of membranes have been treated in detail in $[9,10]$. Below, only basic relations and equations are summarized which are needed in the following considerations.

In the deformed configuration $N$, assumed to be a smooth surface in the space, the equilibrium equations at each point $y \in N$ are

$$
\operatorname{div}_{s} \mathbf{N}+\mathbf{q}=0,
$$

where $\mathbf{N}(y)$ denotes the surface stress tensor (Cauchy type), $\mathbf{q}(y)$ is the surface force and div stands for the surface divergence operator $[9,10]$. Flexible membranes have no bending stiffness and hence they cannot support transverse shear forces. Therefore, $\mathbf{N}(y)$ is the tangential surface tensor (the linear map of the tangent space $T_{y} N$ into itself). Moreover, this tensor is symmetric by virtue of the balance law of moments.

Let $\mathbf{m}$ be the unit normal vector to $N$. The curvature tensor of $N$ is defined by $\mathbf{K}=-\mathbf{P} \operatorname{grad}_{s} \mathbf{m}$, where $\mathbf{P}$ denotes the projection operator (see [9-12]). The differential identity $\mathbf{m} \bullet\left(\operatorname{div}_{s} \mathbf{N}\right)=\mathbf{N} \bullet \mathbf{K}$ may next be used to split the equilibrium equations ( 1 ) into the scalar equation

$$
\mathbf{N} \cdot \mathbf{K}+q=0, \quad q=\mathbf{m} \bullet \mathbf{q},
$$

which represents the equilibrium condition of all forces normal to $N$, and the vector equation in the tangent space $T_{y} N$,

$$
\mathbf{P}\left(\operatorname{div}_{s} \mathbf{N}\right)+\mathbf{P q}=\mathbf{0},
$$

which represent the equilibrium conditions of all forces tangent to $N$ at a given point.

Relative to a fixed reference configuration $M$, the deformation of the membrane is described by a smooth mapping $\chi: M \rightarrow N$ with the tangential deformation gradient $\mathbf{F}(x)=\operatorname{Grad}_{s} \chi(x)$ being the linear map of $T_{x} M$ into $T_{y} N$. This tensor is non-singular and hence it may be written as the product of the surface stretch tensor $\mathbf{U}(x)$ and the tangential rotation tensor $\mathbf{R}(x)$ [9-12]. Then the tangential Cauchy-Green deformation tensor $\mathbf{C}(x)$ and the surface Green strain tensor $\mathbf{E}(x)$ are given by

$$
\mathbf{C}=\mathbf{F}^{\mathrm{T}} \mathbf{F}=\mathbf{U}^{2}, \quad 2 \mathbf{E}=\mathbf{C}-\mathbf{1},
$$

where $1(x)$ denotes the unit tensor on $T_{x} M$. In the description of deformation relative to $M$, it is convenient to introduce the surface stress tensor $\mathbf{S}(x)$ of the second Piola-Kirchhoff type, which is defined by

$$
\mathbf{N}=j^{-1} \mathbf{F S F}^{T}, \quad j=\operatorname{det} \mathbf{F}=\sqrt{\operatorname{det} \mathbf{C}} .
$$

The basic relations and equations summarized above are formally similar to their counterparts in the three-dimensional theory. However, it should be noted that all quantities are defined here on curved surfaces and not in the flat Euclidean space. This is seen from the derivation leading to the equations (2) and (3).

\section{Constitutive modelling damaged membranes}

In the theory of elastic membranes, the constitutive equations relating to surface stresses and strains are typically formulated in terms of a strain energy function which depends on the deformation tensor $\mathbf{C}$ alone. However, inelastic effects are path dependent and their modelling should be based on the thermodynamical theory of irreversible processes. In this paper thermal effects are not considered but the restrictions implied by the principle of irreversibility will be pointed out.

Stress-softening effects are generally attributed to an internal damage of a material. On macroscopic scale, this may be accounted for by a scalar damage variable 
$\alpha(x, t)$ such that $\alpha=0$ in the undamaged (virgin) state of the membrane. Here $t$ is a time-like parameter. According to the general theory of irreversible processes, the complete constitutive model of an inelastic membrane requires that an elastic potential (the free energy function under isothermal conditions) $\Phi$ and the stress tensor $\mathbf{S}$ are given by appropriate constitutive equations in terms of $\mathbf{C}$ and $\alpha$ including possibly their surface and time derivatives. For the purpose of this paper it will suffice to consider the constitutive equations of the form

$$
\boldsymbol{\Phi}=\tilde{\boldsymbol{\Phi}}(\mathbf{C}, \alpha, \dot{\mathbf{C}}), \quad \mathbf{S}=\tilde{\mathbf{S}}(\mathbf{C}, \alpha, \dot{\mathbf{C}})
$$

together with the following evolution equation for the damage variable

$$
\dot{\alpha}=\beta(\mathbf{C}, \alpha, \dot{\mathbf{C}}) .
$$

Here $\widetilde{\Phi}, \widetilde{\mathbf{S}}$ and $\beta$ are given functions of their common arguments. In general, these functions must satisfy the principle of irreversibility (the second law of thermodynamics). From this principle the following results can be derived: (1) the elastic potential $\widetilde{\Phi}$ is independent of $\dot{\mathbf{C}}, \delta_{\dot{C}} \widetilde{\Phi}=0,(2)$ the constitutive equation for the stress is given by

$$
\mathbf{S}=\tilde{\mathbf{S}}(\mathbf{C}, \alpha, \dot{\mathbf{C}})=2 \delta_{\mathbf{C}} \tilde{\mathbf{\Phi}}(\mathbf{C}, \alpha)+\widetilde{\mathbf{S}}_{*}(\mathbf{C}, \alpha, \dot{\mathbf{C}}),
$$

and (3) the following inequality

$$
\widetilde{\mathbf{S}}_{*}(\mathbf{C}, \alpha, \dot{\mathbf{C}}) \cdot \dot{\mathbf{C}}-\left(\delta_{\alpha} \tilde{\Phi}(\mathbf{C}, \alpha)\right) \dot{\alpha} \geq 0
$$

must be satisfied in every deformation process. This inequality simply states that the deformation of a membrane is accompanied by the energy dissipation due to viscous stresses $\widetilde{\mathbf{S}}_{*}(\mathbf{C}, \alpha, \dot{\mathbf{C}})$ and the growth of damage and thus it is an irreversible process.

The constitutive equation (8) is general enough to account for viscous effects. However, although the stresssoftening process depends on deformation history, strain rate dependency often appears to be negligibly small [ 1 , $7,13]$. Therefore, in the remaining part of this paper it will be assumed that the viscous part $\widetilde{\mathbf{S}}_{*}$ of the stress may be neglected. With the assumption that $\widetilde{\mathbf{S}}_{*}(\mathbf{C}, \alpha, \dot{\mathbf{C}})=\mathbf{0}$, the constitutive description of a damaged membrane requires specification of two scalar functions, namely of $\widetilde{\Phi}(\mathbf{C}, \alpha)$ and $\beta(\mathbf{C}, \alpha, \dot{\mathbf{C}})$. Moreover, the assumption that viscous stresses can be neglected requires that $\beta$ is a homogenous function of degree one with respect to $\dot{\mathbf{C}}$. This condition is satisfied if the evolution law (7) is assumed in the special form

$$
\dot{\alpha}=\beta(\mathbf{C}, \alpha, \dot{\mathbf{C}})=\zeta(\mathbf{C}, \xi) \dot{\xi},
$$

where $\zeta(\mathbf{C}, \xi)$ is a given constitutive function and $\xi=\xi(\mathbf{C})$ is the so-called effective or equivalent strain.

The stress-softening effects illustrated in Fig 1 and Fig 2 is a property of a membrane material in which a decrease in stress accompanies an increase in strain. This is a rather special case of the general damage phenomena for which simplifications of the general constitutive equations are possible.

\section{Constitutive modelling stress-softening effect}

If the function $\zeta$ in the kinetic law of damage growth (10) depends on the deformation tensor $\mathbf{C}$ only through the effective strain $\xi=\xi(\mathbf{C})$, then this law takes a simple form $\dot{\alpha}=\zeta(\xi) \dot{\xi}$. Moreover, this relation may be integrated to give the damage variable in the explicit form

$$
\alpha(t)=Z(\xi(t)), \quad Z(\xi(t)) \equiv \int_{\xi(0)}^{\xi(t)} \zeta(\xi(s)) d \xi(s) .
$$

In continuum damage mechanics, various so-called equivalence hypotheses are postulated which considerably simplify the constitutive modelling of damage phenomena. Adopting similar point of view for membranes, it may be assumed that

$$
\widetilde{\Phi}(\mathbf{C}, \alpha)=(1-\alpha) W(\mathbf{C}),
$$

where $W(\mathbf{C})$ denotes a strain energy function of an undamaged membrane. Under this assumption, often referred to as the strain equivalence hypothesis, the stressstrain (8) relation takes the form

$$
\mathbf{S}=(1-\alpha) \delta_{\mathbf{C}} W(\mathbf{C})
$$

with $\alpha$ being given by (11). Within the assumptions leading to (13) and (11), there remains only to specify the damage softening function $Z(\xi)$ and an appropriate damage criteria.

An idealized stress-softening membrane may be considered as an inelastic material having a selective memory of only the maximum previous strain experienced during its deformation. With the effective strain being defined by $\xi(t) \equiv \xi(\mathbf{C}(t))$, the maximum previous strain is $\xi_{\max }(t) \equiv \max _{0 \leq s \leq t} \xi(s)$ with $t$ denoting current time. Moreover, the damage will grow only if $\xi>\xi_{\max }$. Accordingly, the evolution of damage variable $\alpha$ is given by $\alpha=Z(\xi)$ during loading from the undamaged state and $\alpha=Z\left(\xi_{\max }\right)$ during reloading from the state of maximum effective strain.

\section{Isotropic membranes}

Further specifications of various damage models may be obtained if the membrane exhibits certain symmetries in its mechanical properties. The simplest case is that of an isotropy. Elastomeric membranes have this property, in which case the constitutive equation for the stress tensor takes the form (see [10-12])

$$
\mathbf{S}=\widetilde{\mathbf{S}}(\mathbf{C}, \alpha)=h_{0}\left(i_{1}, i_{2}, \alpha\right) \mathbf{1}+h_{1}\left(i_{1}, i_{2}, \alpha\right) \mathbf{C}
$$

independently of various hypothesis considered in the previous chapter. Here $h_{0}$ and $h_{1}$ are given scalar functions of the principal invariants of the deformation tensor $\mathrm{C}$. They are defined by

$$
i_{1}=\operatorname{trC}, \quad i_{2}=\operatorname{det} \mathbf{C} .
$$

The particular form (14) of the stress-strain relations will be obtained in the next chapter, where a more general situation is considered. Here it is worth to note that in the case of isotropic membranes the stress tensor $\mathbf{S}$ and the deformation tensor $\mathbf{C}$ have common principal directions which are determined by two mutually orthogonal 
unit vectors $\mathbf{u}_{1}$ and $\mathbf{u}_{2}$. Denoting by $\lambda_{1}$ and $\lambda_{2}$ the associated principal stretches, the deformation tensor may be written in the canonical form

$$
\mathbf{C}=\lambda_{1}^{2} \mathbf{u}_{1} \otimes \mathbf{u}_{1}+\lambda_{2}^{2} \mathbf{u}_{2} \otimes \mathbf{u}_{2} .
$$

Moreover, in the deformed configuration of the membrane the unit vectors along the principal directions are determined by $\lambda_{1} \mathbf{v}_{1}=\mathbf{F} \mathbf{u}_{1}$ and $\lambda_{2} \mathbf{v}_{2}=\mathbf{F} \mathbf{u}_{2}$. With the use of these relations, the constitutive equation (14) may be rewritten in terms of the Cauchy type stress tensor

$\mathbf{N}=j^{-1}\left\{\lambda_{1}^{2}\left(h_{0}+\lambda_{1}^{2} h_{1}\right) \mathbf{v}_{1} \otimes \mathbf{v}_{1}+\lambda_{2}^{2}\left(h_{0}+\lambda_{2}^{2} h_{1}\right) \mathbf{v}_{2} \otimes \mathbf{v}_{2}\right\} .(17)$

Substituting (17) into (2) and (3), the equilibrium equations are obtained in the form particularly convenient in the analysis of general constitutive equations.

\section{Anisotropic membranes}

The isotropy cannot be assumed in modelling biological membranes. Without this assumption, the elastic potential $\Phi$ must be considered as an arbitrary function of the deformation tensor $\mathbf{C}$ and of the damage variable $\alpha$. Its general form is only restricted by the inequality (9). However, there are experimental evidence that many biological membranes may be modelled as orthotropic ones (see $[2,7,8]$ ). This kind of an anisotropy may be characterised at each point of the membrane by a unit vector a along the preferred direction, usually called the fibre direction. Moreover, in the case of the orthotropic membranes $\mathbf{a}$ and $\mathbf{- a}$ are physically equivalent. Hence, not the vector $\mathbf{a}$ but the tensor $\mathbf{A}=\mathbf{a} \otimes \mathbf{a}$ characterises this class of membranes. Moreover, it follows from the theory of tensor function (see eg [14) that $\Phi$ depends on $\mathbf{C}$ and $\mathbf{A}$ through their common isotropic invariants, ie

$$
\Phi=\Phi\left(i_{1}, i_{2}, i_{3}, \alpha\right),
$$

consisting of the principal invariants (15) of $\mathbf{C}$ and one additional invariant defined by

$$
i_{3}=\mathbf{A} \bullet \mathbf{C}=\mathbf{a} \bullet \mathbf{C a} .
$$

Denoting by $\Phi_{1}, \Phi_{2}, \Phi_{3}$ the derivatives of $\Phi$ with respect to the invariants $\left(i_{1}, i_{2}, i_{3}\right)$, it is easy to shown (see [12]) that the constitutive equation for the stress tensor takes the form

$$
\mathbf{S}=h_{0} \mathbf{1}+h_{1} \mathbf{C}+h_{2} \mathbf{A},
$$

where $h_{0}, h_{1}$ and $h_{2}$ are functions of $\left(i_{1}, i_{2}, i_{3}\right)$ and $\alpha$ given by

$$
h_{0}=2\left(\Phi_{1}+i_{1} \Phi_{2}\right), \quad h_{1}=-2 \Phi_{2}, \quad h_{2}=2 \Phi_{3} .
$$

With $\Phi_{3}=0$, the stress-strain relations (14) follow from (20) and (21). In effect, this is a general procedure for deriving the constitutive equations for other classes of anisotropic membranes, each being completely determined by certain structure tensors. Such tensors has been studied in [14] within the context of plane composites.

\section{Analysis of general models}

The general constitutive model and its various special versions derived in the previous chapters may be used together with the equilibrium equations (2) and (3) in the analysis of stress-softening effects in membranes. However, due to path dependent nature of the damage process, a numerical integration algorithm is required for the evaluation of stresses and damage evolution throughout the entire deformation history. The problem is simplified considerably if the evolution of the damage variable is given explicitly by the relation (11). In the particular case of the inflation of spherical membranes, the resulting equation follows from (17) and (2) while the equilibrium equation (3) is trivially satisfied. The problem is thus reduced to a single algebraic for the radial stretch.

One of the conceptual differences between problems of the classical linear elasticity and those of the finite strain deformation of membranes is that the specification of the form of constitutive relations is generally part of problem in the theory of membranes whereas in the classical theory one needs only to specify the material constants occurring in the generalized Hooke's law. This is not a trivial difficulty since the various natural and synthetic membranes which can sustain large elastic and inelastic deformations each behave somewhat differently.

In the case of elastic membranes, the problem is reduced to the identification of material parameters for the assumed form of a strain energy function $W(\mathbf{C})$. This is still a non-trivial task due to limited number of available experimental results and difficulties in the interpretation of the results obtained by different authors. This is illustrated in Fig 3. The presented analytical solutions were obtained for the strain energy function $W(\mathbf{C})$ derived from the three-dimensional constitutive equation of Hart-Smith [15] for rubber-like materials using the method [12].

In the analysis of damage phenomena in general and of the stress-softening effects in particular, the problem of identification of constitutive equations is even more difficult. Even if the strain equivalence hypothesis (12) is adopted and if a particular form of the strain energy function $W(\mathbf{C})$ is assumed, there still remains to specify the effective strain $\xi$ and the softening function $Z(\xi)$. In general, a growth of damage in stress-softening membranes is only delimited by the inequality (9). Under the

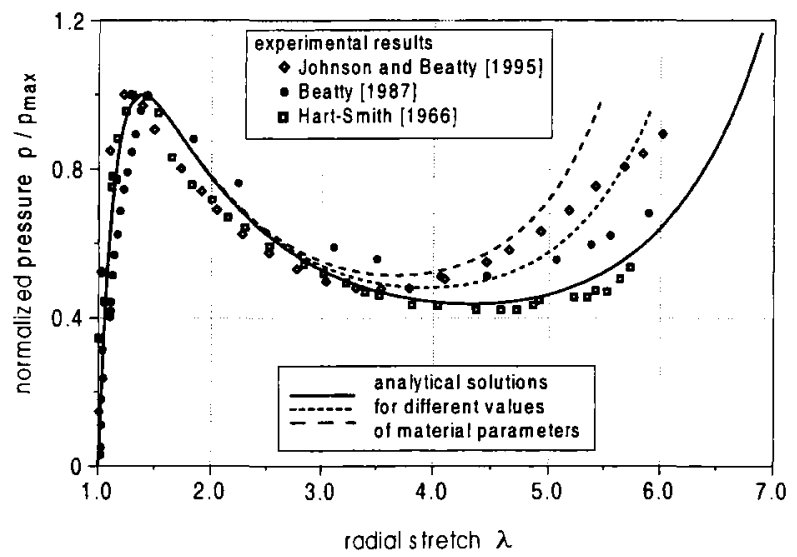

Fig 3. Experimental results and analytical solutions for a membrane modelled as Hart-Smith material 


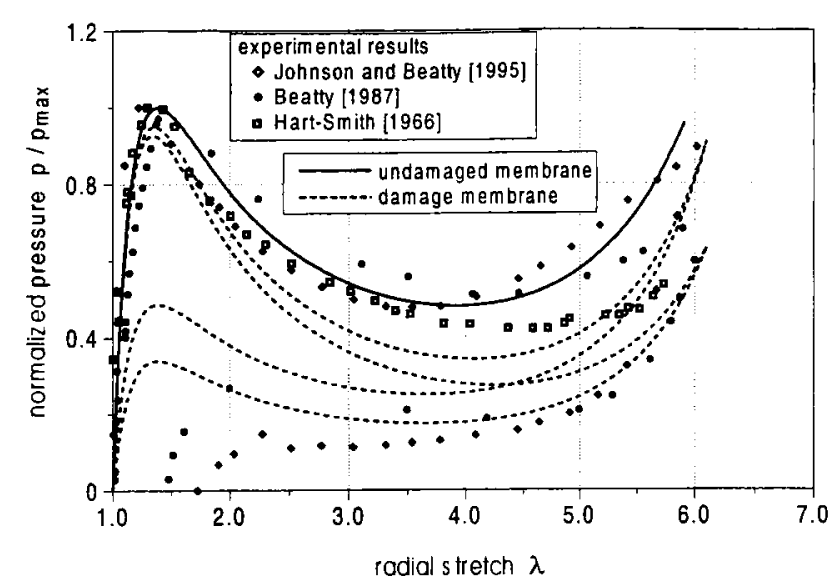

Fig 4. Parametric study of softening effects

assumption (12), this inequality takes the form $W(\mathbf{C}) \dot{\alpha} \geq 0$ and since $W(\mathbf{C}) \geq 0$ by virtue of the basic results in the non-linear elasticity, we must have $\dot{\alpha} \geq 0$. In effect, the softening in the membrane response increases with increasing values of the effective strain $\xi$. Hence, $Z(\xi)$ must be a monotonously increasing functions of $\xi$. A simple example of such a function is (cf. [13])

$$
Z(\xi)=1-\mu \exp \left(-\eta\left(\xi-\xi_{0}\right)\right)
$$

where $\mu$ and $\eta$ are parameters characterising growth of damage and $\xi_{0}$ is an initial damage threshold.

In general, $Z(\xi)$ is given by (11) under the assumption that the constitutive function $\zeta$ appearing in the kinetic law of damage growth (10) depends on $\mathbf{C}$ only through the effective strain $\xi=\xi(\mathbf{C})$. It follows then that a specific form of the softening function $Z(\xi)$ is essentially independent of possible material symmetries. In contrast, the effective strain is a function of the deformation tensor $\mathbf{C}$ and thus its specific form should respect particular material symmetries.

In the case of isotropic membranes, the effective strain may be defined as an isotropic function of $\mathbf{C}$, ie as a given function $\xi=\xi\left(i_{1}, i_{2}\right)$ of the invariants (15). For orthotropic membranes, the corresponding general definition would be $\xi=\xi\left(i_{1}, i_{2}, i_{2}\right)$. For illustration only, a simple form of such a function may be assumed

$$
\xi=\sqrt{i_{1}^{2}-2 i_{2}} \text {. }
$$

With the use of the effective strain defined by (23), the solutions for different values of the parameters appearing in the softening function (22) are shown in Fig 4. It can be seen from these simple calculations that various types of stress-softening can be modelled by varying only the parameters in the assumed damage softening function. However, a complete study of this problem is beyond the aim of this paper.

\section{Closing remarks}

While three-dimensional constitutive equations of continuum mechanics may be specified for membranes and thus they may be used to analyse their inelastic be- haviour, for polymeric and biological membranes most of the available experimental data have been obtained in biaxial experiments $[1-4,8,15]$. Although biaxial tests alone are not sufficient to derive a three-dimensional stress-strain relationships, for membranes they suffice to yield the two-dimensional constitutive equations relating to the surfaces stresses and surface strains. The general results derived in this paper provide the conceptual framework within which specific forms of constitutive equations for membranes may be sought entirely independently of any three-dimensional theory.

Acknowledgement. This research was partially supported by the Polish State Committee for Scientific Research under grant KBN 7 T07A 02116.

\section{References}

1. Johnson M. A., Beatty M. F. The Mullins effect in equibiaxial extension and its influence on the inflation of a balloon. Int. J. Engng Sci., 33, 1995, p. 223-245.

2. Sacks M. S. Biaxial mechanical evaluation of planar biological materials. J. Elasticity, 61, 2000, p. 199-246.

3. Rand R. P. Mechanical properties of the red cell membrane, II. Viscoelastic breakdown of the membrane. Biophysical J., 4, 1964, p. 303-316.

4. Hochmuth R. M., Hampel W. L. Surface elasticity and visco-elasticity of red cell membranes. J. Rheology, 23, 1979, p. $669-680$.

5. Wineman A. On axisymmetric deformations of nonlinear viscoelastic membranes. J. Non-Newtonian Fluid Mech., 4, 1978, p. 249-260.

6. Feng W. W. Viscoelastic behavior of elastomeric membranes. ASCE J. Appl. Mech., 59, 1992, p. S29-S34.

7. Fung Y. C. Biomechanics. Mechanical properties of living tissues. New York: Springer-Verlag, 1981.

8. Sacks M. S. A structural constitutive model for chemically treated planar tissues under biaxial loading. Comput. Mech., 26, 2000, p. 243-249.

9. Gurtin M. E., Murdoch A. I. A continuum theory of elastic material surfaces. Arch. Rat. Mech. Anal., 57, 1975, p. 291-323.

10. Murdoch A. I., Cohen H. Symmetry considerations for material surfaces. Arch. Rat. Mech. Anal., 72, 1979, p. 61-98.

11. Kazakevičiūtè-Makovska R. Structure of constitutive relations for isotropic elastic membranes with voids. Statyba, Vol VII, No 1. Vilnius: Technika, 2001, p. 23-28.

12. Kazakevičiūtè-Makovska R. Nonlinear response functions for transversely isotropic elastic membranes. Statyba, Vol VII, No 5. Vilnius: Technika, 2001, p. 345-351.

13. Beatty M. F., Krishnaswamy $S$. The Mullins effect in equibiaxial deformation. Z. angew. Math. Phys., 51, 2000, p. $984-1016$.

14. Zheng Q.-S., Betten J. The formulation of constitutive equations for fibre-reinforced composites in plae problems: Part II. Arch. Appl. Mech., 65, 1995, p. 161-177.

15. Hart-Smith L. J. Elasticity parameters for finite deformations of rubber-like materials. Z. angew. Math. Phys., 17, 1966, p. 608-626. 